\title{
Ultrasonic measurements and modelling of attenuation and phase velocity in pulp suspensions
}

\author{
Jan Niemi*, Yvonne Aitomäki and Torbjörn Löfqvist \\ Department of Computer Science and \\ Electrical Engineering \\ Lulea University of Technology, Sweden \\ *Email: jan.niemi@ltu.se
}

\begin{abstract}
In the manufacturing process of paper the mass fraction and material properties of the fibres in the pulp suspension are important for the quality of the finished product. This study presents two different methods of pulp characterisation. The first is based on phase velocity, which we use to investigate the composition of the pulp. Here a method is presented where the optimal number of circular shifts within the sampling window of the signal is determined which gives, in a weakly dispersive medium, a continuous phase spectrum and minimizes the likelihood of discontinuities within the bandwidth. Hence, the ambiguity in phase unwrapping is avoided. The results from phase velocity measurements show that the phase velocity weakly increases with increasing amount of fines in the suspension. The dispersion is caused by the fibres and it correlates with fibre mass fraction. The second method is based on attenuation and is used to characterise the wood fibres. The results of the attenuation experiments show that it is possible to inversely calculate wood fibre properties by fitting the model to the experimental data, if the fibre diameter distribution is known. However, the accuracy of these calculation is difficult to determined and more work in this area is required.
\end{abstract}

\section{INTRODUCTION}

In the manufacturing process of paper the mass fraction and material properties of the fibres in the pulp suspension are important for the quality of the finished product. When using recycled paper, fibres with unknown and varying material properties enter the process. Therefore, there is an increasing demand for methods of on-line characterisation of the pulp suspension as well as the fibres in suspension.

This study presents two different methods of pulp characterisation. The first is based on phase velocity, which we use to investigate the composition of the pulp. The second is based on attenuation and is used to characterise the wood fibres.

In the first method, we investigate how the phase velocity changes with different mass fractions of fibres and fines. To determine the phase velocity, a method is proposed based on a method by [1], where the an echo is circularly shifted an optimal number of samples.

In the second method, to be able to characterise the wood fibres, we use an analytical model which relates the material properties of saturated fibres to the attenuation. We then aim to solve the inverse problem of identifying which values result in the best fit of the model to the attenuation values calculated from experiments.

\section{Phase Velocity}

\section{A. Theory and experiments}

When determining the phase velocity from pulse-echo measurements, one encounters the problem of performing a correct phase unwrapping. The problem is well known and has been addressed in earlier investigations, for instance [2]. The problem arises when the phase velocity is calculated from the phase spectra of a the Fourier transform of each of the two echoes. In this study, we propose a method, termed Minimum Phase Angle (MPA), that determines an optimal number of circular shifts to the windowed signal which results in a continuous phase spectrum and minimizes the likelihood of discontinuities within the bandwidth. Therefore the ambiguity in the phase unwrapping is avoided. To experimentally test the method experiments were performed in pulp fibre suspensions, which are weakly dispersive. The experiments were carried out using the pulse-echo technique in a custom designed test cell. A schematical view of the measurement cell used in this study is shown in Fig. 1.

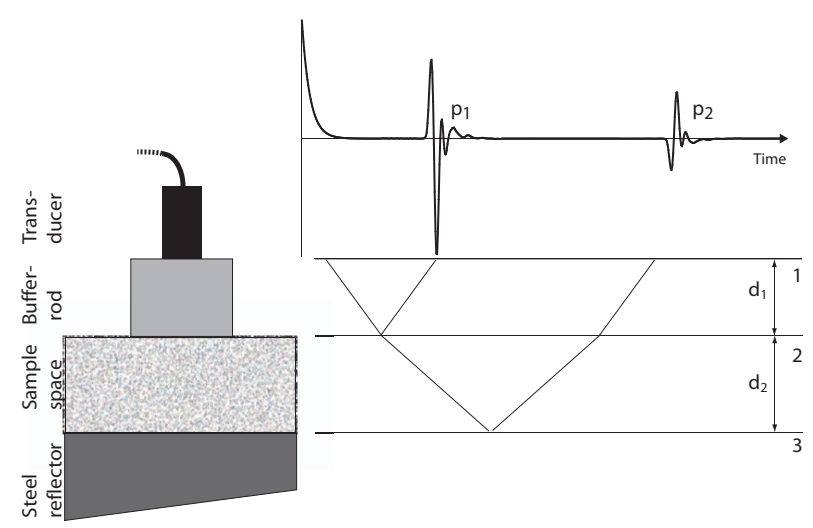

Fig. 1. Lattice diagram of the pulse-echo measurement system used this study

The echoes from the interfaces depend on the initial pulse pressure amplitude $p_{0}(t)$ emitted from the transducer and the reflection and transmission coefficients of the different interfaces. For simplicity, we omit the reflection and transmission coefficients and the attenuation. With these assumptions, the echoes from the interfaces between the buffer rod/suspension 
and suspension/steel reflector are

$$
\begin{aligned}
& P_{1}(\omega)=P_{0}(\omega) e^{-2 j \frac{\omega}{c_{1}} d_{1}}=P_{0}(\omega) e^{-j \varphi_{1}(\omega)} \\
& P_{2}(\omega)=P_{0}(\omega) e^{-2 j \omega\left(\frac{d_{1}}{c_{1}}+\frac{d_{2}}{c_{2}}\right)}=P_{0}(\omega) e^{-j \varphi_{2}(\omega)}
\end{aligned}
$$

where $P_{1}(\omega)$ and $P_{2}(\omega)$ are the Fourier transform of the echoes $p_{1}(t)$ and $p_{2}(t)$, respectively. $d_{1}$ and $d_{2}$ are the distance in respective medium. The factor 2 above comes from that the fact the pulse is travelling back and forth through the medium. $c_{1}$ and $c_{2}$ are the velocities in the buffer rod and pulp suspension, respectively. $P_{0}(\omega)$ is the Fourier transform of the initial emitted pulse from the transducer. Note that echo $p_{1}(t)$ has an extra phase shift of $\pi$ compared to $p_{2}(t)$.

\section{B. The method of minimum phase angle}

To reduce the ambiguity in the phase unwrapping the following method is proposed.

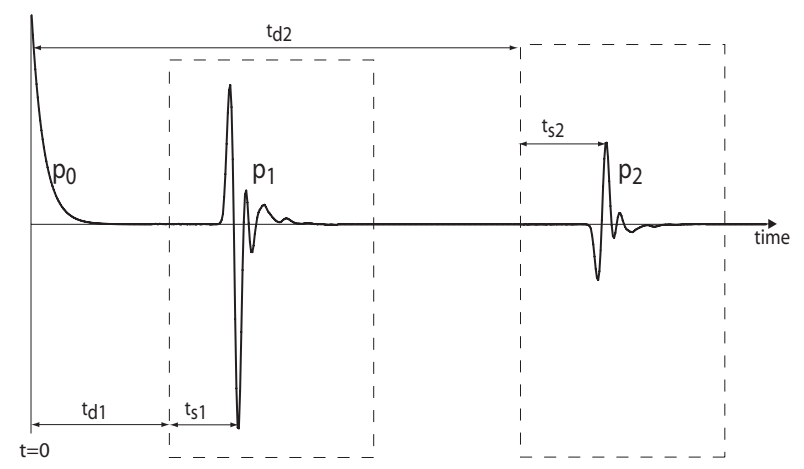

Fig. 2. The received signal with illustrations of the time delays and time shifts

The sampled signal is divided into two sampling windows with time delays $t_{d}=n_{d} / f_{s}$ shown in Fig. 2 where $n_{d}$ is the number of samples that the sampling window is delayed and $f_{s}$ is the sampling frequency. Within each sampling window the signal is circularly shifted sample by sample. At each shift, a calculation of the phase angle is carried out as

$$
P A=\frac{1}{f_{2}-f_{1}} \sum_{m=f_{1}}^{f_{2}} \varphi_{m}^{2}
$$

where $\varphi_{m}$ is the phase spectrum within the frequency bandwidth of $f_{1}<m<f_{2}$ of the shifted sample. Thereafter a sign shift of the echo is carried out, representing a phase shift of $\pi$, and again circularly shifted and calculated with the same method. The results are compared and the circularly shifted sample that gives a phase spectrum without discontinuities and minimum value of $P A$ is then chosen. The outcome is then the optimal time shift of $t_{s}=n_{s} / f_{s}$ where $n_{s}$ is the number of samples the signal is shifted.

This results in two time delays, the time delay from the sampling window and time delay from the circular shift within the window. This gives the phase spectrum for the respective echoes as

$$
\begin{aligned}
& \varphi_{1}(\omega)=\phi_{1}(\omega)+\omega\left(t_{d 1}+t_{s 1}\right) \\
& \varphi_{2}(\omega)=\phi_{2}(\omega)+\omega\left(t_{d 2}+t_{s 2}\right)
\end{aligned}
$$

where $\phi_{1}$ and $\phi_{2}$ are the respective phase spectra of the circularly shifted signal. $t_{d 1}$ and $t_{d 2}$ are the time delay for respective sampling window. $t_{s 1}$ and $t_{s 2}$ are the circular shift within respective sampling window. An illustration of how $P A$ changes when echo $p_{1}(t)$ is circularly shifted within the sampling window is shown in Fig. 3. In this example, the minimum of $P A$ is found when $p_{1}(t)$ is shifted 100 samples to the left and inverted. Fig.3(c) shows the phase spectrum at the minimum of $P A$ for $p_{1}(t)$ and $-p_{1}(t)$. We can see that the inverted signal has a phase spectrum with the minimum likelihood of a discontinuity being present in the spectrum.

The phase velocity for a pulp suspension sample can be expressed using (1)-(2) and (4)-(5) as

$$
c_{2}(\omega)=\frac{\omega 2 d_{2}}{\omega\left(t_{2}-t_{1}\right)+\theta(\omega)-\theta_{d}(\omega)+m \pi}
$$

where $t_{1}=t_{d 1}+t_{s 1}, t_{2}=t_{d 2}+t_{s 2}, \theta(\omega)=\phi_{2}(\omega)-\phi_{1}(\omega)$, $\theta_{d}(\omega)$ is the phase difference due to diffraction [3] and $m$ is a correction term if a phase shift of $\pi$ is added by the proposed method. In this study $m=0$ if the proposed method inverts $p_{1}(t)$ to compensate for the extra phase shift that occurred, $m=1$ if $p_{1}(t)$ and $p_{2}(t)$ are not inverted and $m=-1$ if both $p_{1}(t)$ and $p_{2}(t)$ are inverted.

\section{Experiment}

The experimental setup consists of a broadband transducer with a centre frequency of $10 \mathrm{MHz}$ (V311), manufactured by Panametrics, Waltham, MA, USA. A pulser/receiver 5072PR from Panametrics was used to excite the transducer and amplify the received signal. The signal was then digitized using a CompuScope 14100 oscilloscope card, by Gage Applied Technologies Inc., Lachine, QC Canada, with 14-bit resolution and a sampling rate of $100 \mathrm{MHz}$. All data was stored in a computer for off-line analysis. The resulting time-domain waveforms were calculated off-line using the average of 100 sampled waveforms to reduce random noise. Before the averaging process, the sampled waveforms are aligned to reduce timing jitter by employing a method proposed by [4]. A digital thermometer F250, by Automatic Systems Laboratories LTD, England, monitored the temperature both in the suspension under test and in the room. The temperature in the pulp suspensions under test was $20.0 \pm 0.2^{\circ} \mathrm{C}$. The pulp suspensions was carefully poured into the measurement cell and thereafter stirred slowly to remove air bubbles from the suspension. An illustration of the measurement cell is shown in Fig. 1 and is described in detail in [5]. To accurately determine the distance $d_{2}$ in the cell, pure, distilled water was used as a reference since it has a well known relationship between speed of sound and temperature, see [6]. Using the temperature of the calibration fluid and a cross-correlation technique to determine 


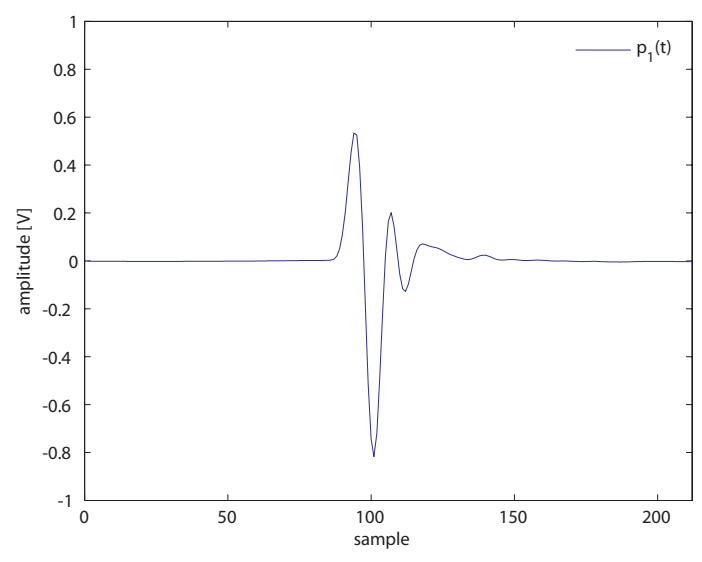

(a)

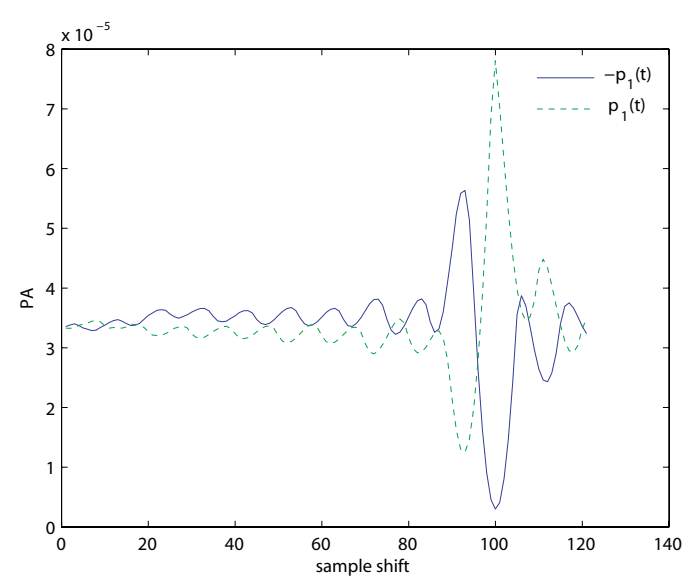

(b)

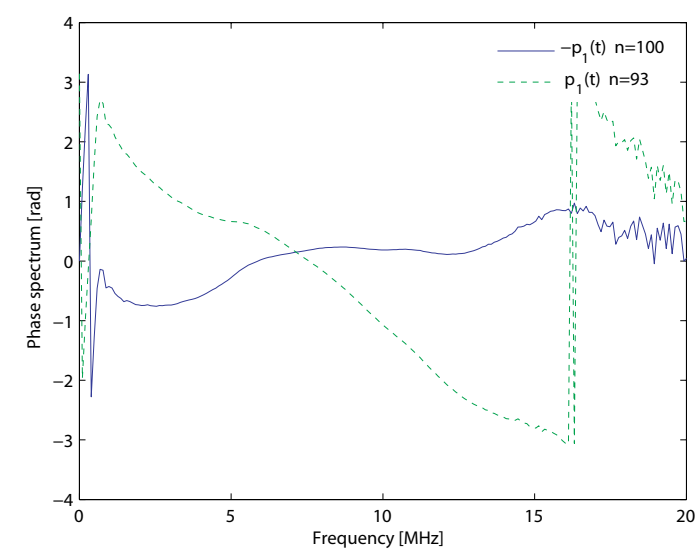

(c)

Fig. 3. (a) shows echo $p_{1}(t)$. (b) shows the results of $p a(m)$ from $p_{1}(t)$ and when $p_{1}(t)$ is inverted. (c) shows the phase spectrum of $p_{1}(t)$ and $-p_{1}(t)$ when the echoes are shifted 93 and 100 samples respectively, i.e at respective minimum of $p a$

the time-of-flight for an ultrasonic pulse, the distance $d_{2}$ was found to be $0.03010 \pm 0.00004 \mathrm{~m}$.
The pulp samples used in this study were produced from thermo-mechanical pulp (TMP). The TMP was fractionated by Bauer-McNett fractionator according to SCAN-standard 6:69 [7]. This process separated the pulp into two fractions; a fibre fraction and a fines fraction. The fibre fraction consists of fibres that passed the 48 wire mesh, resulting in fibre lengths that vary between $1-3 \mathrm{~mm}$ and diameter of $20-50 \mu \mathrm{m}$. The fines were obtained by passing the pulp through the 200 wire mesh and then subsequently filtered through 400 wire mesh. They have a length of 30-74 $\mu \mathrm{m}$ and a diameter of a few $\mu \mathrm{m}$. Both the fines and fibre size distributions were analyzed using a Kajaani Fiberlab instrument, Metso Corporation, Finland. From these fractions, a set of samples were made by mixing fibres and fines at predetermined ratios between $0-1.0 \%$ by mass.

\section{Results}

The results from the phase velocity measurement for suspensions containing only fines is shown in Fig.4. The figure shows that the velocity dispersion is small within the bandwidth of the ultrasonic pulse, $1.8-10 \mathrm{MHz}$, and that the phase velocity increases with increasing amount of fines in the suspension.The uncertainty in the measurement is \pm 0.3 $\mathrm{m} / \mathrm{s}$ based on \pm 2 standard deviations.

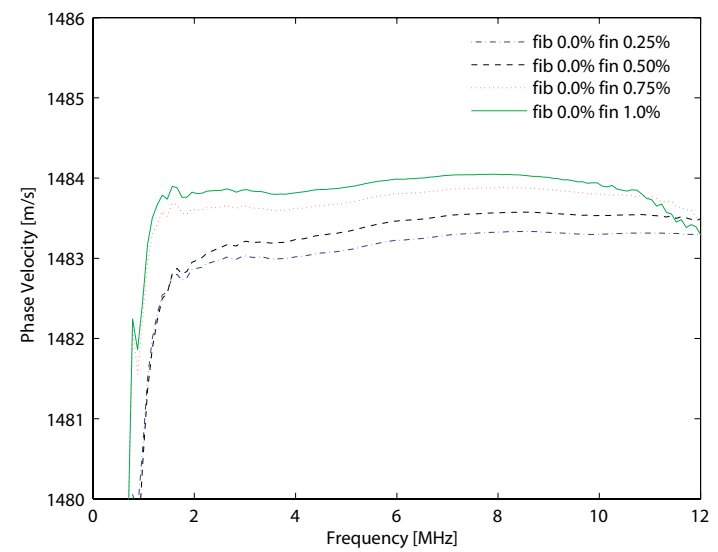

Fig. 4. Phase velocity in pulp suspension containing fines and no fibres

Fig. 5, shows measurements of phase velocity for samples where the mass faction of fibres are the same as for fines. In this case, the velocity dispersion is noticeable and it is seen to correlate well with mass fraction, giving higher velocity dispersion with higher mass fraction. As an example, the velocity for $1.0 \%$ mass fraction changes from the lowest below $4 \mathrm{MHz}$ to be the highest above.

\section{ATTENUATION}

\section{A. Theory}

The model is based on calculating the energy loss in the scatter wave from an infinitely long, viscoelastic, isotropic, cylindrical fibre. It is described in full in [8]. In [8], the modelled attenuation agrees well with experimental results of 


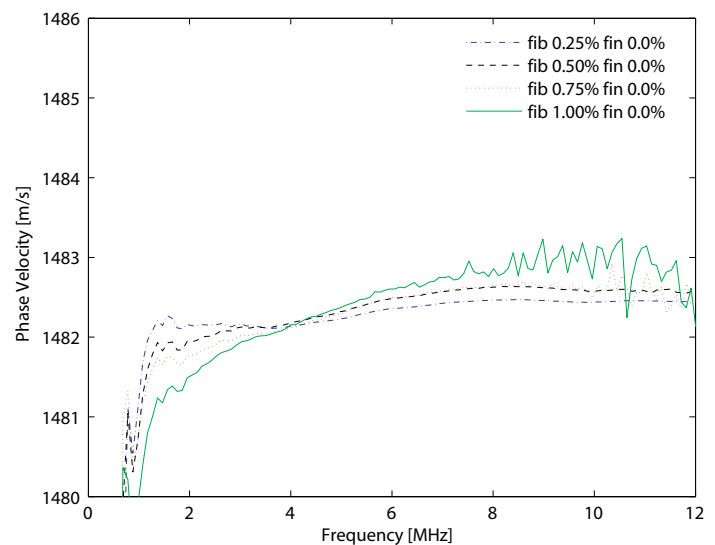

Fig. 5. Phase velocity in pulp suspension containing fibres and no fines

synthetic fibres such as nylon and shows localised extrema in the frequency response of attenuation. These peaks in frequency response of the attenuation are thought to be at the vibrational modes of the fibres. Although the locations of these peaks depend largely on the diameter of the saturated fibres, they also depend on their material properties.

In nylon, the locations of the peaks are more repeatable and hence more reliable than the measurement of the attenuation itself which varies due to the inhomogeneous nature of the suspension. The repeatability of the frequency at which these peaks occur and the fact that changes in diameter, Poisson's Ratio, Young's Modulus and the density of the fibre produce different effects on the shape of the modelled frequency response of the attenuation, means that the suspension can be better characterised if these extrema can be located.

The aim is, therefore, to determine if localised extrema also exist in the frequency response of the attenuation of wood fibres. This would allow us to solve the inverse problem with less ambiguity than attempting to solve the inverse problem from a simple curve.

The model shows the attenuation in the frequency range $1 \mathrm{Mhz}$ to $25 \mathrm{MHz}$ to be very sensitive to fibre diameter. Using an average fibre diameter of $40 \mu \mathrm{m}$ localised maxima in attenuation were predicted to be between $5 \mathrm{MHz}$ to $10 \mathrm{MHz}$. However, the wood fibres in paper pulp have different diameters hence the diameter distribution of the wood samples is required to produce expected attenuation.

\section{B. Experiment}

The same measurement cell and paper pulp samples were used as in the phase velocity experiments. As the diameter of wood fibres are smaller than nylon the peaks are expected to appear at a higher frequency. Hence a $30 \mathrm{MHz}$ transducer (Panametric V333) was used and the signal digitised by a CompuScope 102G oscilloscope card (Gage Applied Technologies, Inc Lachine QC Canada) with a 10-bit resolution and a sample rate of $2 \mathrm{GHz}$. The distance the signal travelled was calculated using the method previously described. Since the initial echo from the buffer rod could not be easily identified, the attenuation in the sample was obtained using pure, distilled water as reference giving,

$$
\alpha_{s}(f)=\alpha_{w}(f)+\frac{1}{2 d_{2}} \ln \left(\frac{\left|P_{w}(f)\right|}{\left|P_{s}(f)\right|}\right)
$$

where $\alpha_{s}$ is the attenuation in the sample, $d_{2}$ is the distance travelled by the signal, $P_{s}(f)$ is the amplitude in the frequency domain of the second echo in the sample and $P_{w}(f)$ is the amplitude in the frequency domain of the second echo in water. $\alpha_{w}(f)$ is the attenuation of water [9] and is assumed to be

$$
\alpha_{w}(f)=25 \cdot 10^{-15} f^{2}
$$

C. Results

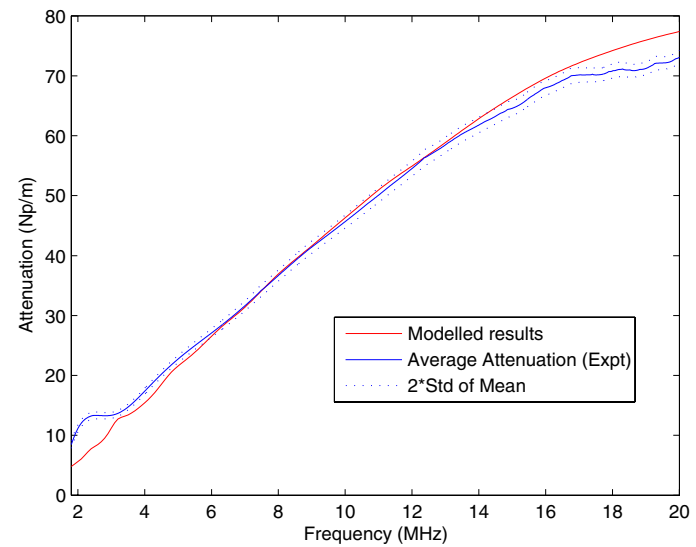

Fig. 6. Comparison of the modelled attenuation to the measured attenuation for $0.5 \%$ concentration of wood fibres. Plot of the mean and 2x STD of the mean for 300 readings. The following material properties for saturated wood fibres were used in the model: density $=1500 \mathrm{~kg} / \mathrm{m}^{3}$, Poisson ratio $=0.45$ speed of sound $=1050 \mathrm{~m} / \mathrm{s}$ and loss tangent $=0.2$. The properties of water were assumed to be: density $=996 \mathrm{~kg} / \mathrm{m}^{3}$, speed of sound $=1490 \mathrm{~m} / \mathrm{s}$, and viscosity $=9.4 \cdot 10^{-4} \mathrm{~Pa} \cdot s$

Figure 6 is a comparison of the experimentally calculated average attenuation and the modelled attenuation. Good agreement has been obtained by adjusting the saturated wood fibre properties. The maximum at $2.2 \mathrm{MHz}$ in the experimental results is not reflected in the model results though a small maximum does exists at $2.4 \mathrm{MHz}$ and a slightly large one at $3.2 \mathrm{MHz}$, corresponding to fibres with diameters of $60 \mu \mathrm{m}$ and $40 \mu \mathrm{m}$ respectively. This lack of agreement could be because the model is for a fibre that is continuous over its cross section. As wood fibres are hollow the vibrational modes, which are believed to be causing the maxima, will not occur at the frequencies predicted by the model. However, it may also be due differences between the distribution of the diameters in this particular sample and that used in the size analysis. The peak could also be an experimental artifact. The sensitivity of the modelled attenuation on the fibre diameter, means the distribution of the diameters is the dominant factor in determining the material properties of the suspended fibres. With unknown fibre diameter distributions, there is a greater 
possibility of determining the diameters of the fibres if the local extrema in the frequency response of the attenuation are clear. However, the superposition of maxima and minima of one wood fibre diameter with other fibres of a different diameters results in a the smoothing of the signal, masking the effects of the individual fibres. The featurelessness of the curve means that the calculation of the material properties from the experimental measured attenuation is ambiguous. Combinations of different values can give similar results.

\section{CONCLUSION}

In this study we have considered measurements of phase velocity and attenuation in pulp suspensions. The proposed method to calculate phase velocity avoids the ambiguity with phase unwrapping if the medium is weakly dispersive. The result shows that the phase velocity increases with increasing amount of fines in the suspension. The dispersion is caused by the fibres and it correlates with fibre mass fraction.

The results of the attenuation experiments show that it is possible to inversely calculate wood fibre properties by fitting the model to the experimental data, if the fibre diameter distribution is known. However, the accuracy of these calculation is difficult to determined and more work in this area is required.

\section{FURTHER WORK}

The proposed minimum phase angle, or MPA, method has to be tested in cases when the phase velocity is highly dispersive.

The peak in the experimental results of the frequency response of attenuation at $2.2 \mathrm{MHz}$ needs further investigation. Experiments on synthetic fibres are being carried out to explore the effect of hollow compared to solid fibres. If these effects are significant, further development of the model is required to take this into account.

\section{REFERENCES}

[1] P. He, "Experimental verification of models for determining dispersion from attenuation," IEEE Transactions on Ultrasonics, Ferroelectrics, and Frequency Control, vol. 46, no. 3, pp. 706-714, 1999.

[2] J. Tribolet, "A new phase unwrapping algorithm," IEEE Transactions on Acoustics, Speech, and Signal Processing, vol. 25, no. 2, pp. 170-177, 1977.

[3] P. Rogers and A. Van Buren, "An exact expression for the lommel diffraction correction integral," Journal of the Acoustical Society of America, vol. 55, no. 4, pp. $724-8,1974$.

[4] A. Grennberg and M. Sandell, "Estimation of subsample time delay differences in narrowband ultrasonic echoes using the hilbert transform correlation," IEEE Transactions on Ultrasonics, Ferroelectrics, and Frequency Control, vol. 41, no. 5, pp. 588-595, 1994.

[5] T. Lofqvist, "Ultrasonic wave attenuation and phase velocity in a paperfibre suspension," in Proc.IEEE Ultrasonics Symposium, vol. 1, 1997, pp. $841-844$.

[6] N. Bilaniuk and G. S. K. Wong, "Speed of sound in pure water as a function of temperature," The Journal of the Acoustical Society of America, vol. 93, no. 4, pp. 2306-2306, 1993.

[7] SCAN-M 6:69, Fibre fractionation of mechnical pulp in the McNett apparatus, Scandinavian Pulp, Paper and Board Testing Committee, Stockholm.

[8] Y. Aitomäki, "Measuring suspended fibre material properties by modelling ultrasound attenuation," in Mathematical Modelling of Wave Phenomena, 2005.

[9] L. Kinsler, A. Frey, A. Coppens, and J. Sanders, Fundamentals of Acoustics, 4th ed. John Wiley and Sons Inc., 2000. 